\title{
Correlation between p-GaN growth environment with electrical and optical properties of blue LEDs
}

\author{
Modestas Zulonas $^{* a}$, Ilya E. Titkov ${ }^{\mathrm{a}}$, Amit Yadav ${ }^{\mathrm{a}}$, Ksenia A. Fedorova ${ }^{\mathrm{a}}$, Andrey F. Tsatsulnikov ${ }^{\mathrm{b}}$, \\ Wsevolod V. Lundin ${ }^{b}$, Alexey V. Sakharov ${ }^{b}$, Thomas Slight ${ }^{\mathrm{c}}$, Wyn Meredith ${ }^{\mathrm{c}}$ and Edik U. Rafailov ${ }^{\mathrm{a}}$ \\ ${ }^{a}$ Optoelectronics and Biomedical Photonics Group, Aston University, Birmingham, B4 7ET, UK; \\ ${ }^{b}$ Ioffe Physico-Technical Institute, 26 Polytechnicheskaya Str., St. Petersburg, 194021, Russia; \\ ${ }^{\mathrm{c} C}$ Compound Semiconductor Technologies Global Ltd, (CSTG), 4 Stanley Boulevard, Hamilton \\ International Technology Park, Hamilton, G72 0BN, Scotland
}

\begin{abstract}
Two blue $(450 \mathrm{~nm})$ light-emitting diodes (LED), which only differ in top $\mathrm{p}$-GaN layer growth conditions, were comparatively investigated. I-V, C-V, TLM, Electroluminescence (EL) and Photoluminescence (PL) techniques were applied to clarify a correlation between MOCVD carrier gas and internal properties. The A-structure grown in the pure $\mathrm{N}_{2}$ environment demonstrated better parameters than the B-structure grown in the $\mathrm{N}_{2} / \mathrm{H}_{2}(1: 1)$ gas mixture. The mixed growth atmosphere leaded to an increase of sheet resistances of p-GaN layer. EL and PL measurements confirmed the advantage of the pure $\mathrm{N}_{2}$ utilization, and $\mathrm{C}\left(\mathrm{V}_{\mathrm{R}}\right)$ measurement pointed the increase of static charge concentration near the $\mathrm{p}$-GaN interface in the B structure.
\end{abstract}

Keywords: Light emitting diode, epi layer, magnesium, p-GaN, MOCVD, capacitance-voltage

\section{INTRODUCTION}

Nowadays Solid State Lighting (SSL), especially light emitting diodes (LED) are the most promising lighting technology that have huge potential over common lighting sources for illumination, like compact fluorescence lamps (CFL), halogen lamps, expiring incandescence bulbs, and etc [1]. The LEDs have advantage of energy saving benefits, efficiency, environment friendly, lifespan, color qualities-tuning, compactness and ruggedness. Nevertheless there are many white color LED bulbs and luminaries on the market already, but there still is a potential of ultimately perfect efficiency white color LED. One of the European Commission project NEWLED (FP7-318388) targets towards developing high brightness and high efficacies white color LEDs for illumination. The aim is to achieve white color light source of CRI $>95 \%$ and light output of $200 \mathrm{~lm} / \mathrm{W}$, while possibly remaining or reducing the cost of the LED device itself [2].

One of the most common methods to produce white color from LED device is to use the Phosphor Conversion (PC) technology [3]. The technique were initially developed by OSRAM Opto Semiconductors GmbH and Nichia corp., and are currently the most widely used type of LED. The white LED light is obtained by mixing blue light emitted by GaNbased LEDs and phosphor converted yellow (yellow-red) light. The advantages of high efficiency, low dependence of the emission spectrum on drive current and operation temperature and simplicity and low cost of a simple single-chip device for a white light emitter has led these white LEDs to dominate the market, with a commercially available efficiency of over $150 \mathrm{~lm} / \mathrm{W}$ and reaching reported values of over $200 \mathrm{~lm} / \mathrm{W}$ for laboratory samples. The theoretical limit for efficiency of this type of LEDs is 280-300 lm/W (for 100\% WPE of blue LED), depending on the correlated color temperature (CCT).

The disadvantages of the approach mainly arise from the use of phosphor-based conversion materials in the LED leading to reduced emission efficiency and increased heat generation due to inevitable Stokes losses in the phosphor and limited lifetime of phosphor and capping layer materials due to the short wavelength component of the blue radiation component. Furthermore, only very limited adjustment of color quality (color temperature, color rendering) is possible. These LEDs also require rare earth materials which are a critical supply/demand issue the impact of which is beginning

Light-Emitting Diodes: Materials, Devices, and Applications for Solid State Lighting XX, edited by Heonsu Jeon,

Li-Wei Tu, Michael R. Krames, Martin Strassburg, Proc. of SPIE Vol. 9768, 97680N · @ 2016 SPIE CCC code: $0277-786 X / 16 / \$ 18 \cdot$ doi: $10.1117 / 12.2211046$ 
to be felt by the EU electronics industry. China controls up to $97 \%$ t of the world's supply of rare earth metals and phosphor prices have been spiraling.

In phosphor-based LEDs warm and cold tones are generated by changing the intensity balance of blue and yellowishorange lines, whereas natural sunlight is characterized by changing of a spectral radiation position generally. This difference can lead to suppression of the synthesis of sleep hormone melatonin in a human body and have a negative impact on human health.

The first step towards producing white color LED is to develop efficient blue color LED [4].

The growth of GaN:Mg is a complex task and by publications it is not clear which type of carrier gas is preferable. In [5] mentioned that $\mathrm{Mg}$ incorporation is higher for $\mathrm{N}_{2}$ carrier gas, but the efficiency of $\mathrm{Mg}$ activation is higher when $\mathrm{H}_{2}$ is used as carrier gas. In [6] mentioned that hydrogen in the reactor ambient can results in surface morphology degradation. Moreover, it was found that high temperature $\left(1100^{\circ} \mathrm{C}\right)$ growth shows better results for $\mathrm{N}_{2}: \mathrm{H}_{2}=1: 1$ while for lower temperature $\left(980^{\circ} \mathrm{C}\right) \mathrm{N}_{2}$ carrier gas is preferable. For real LED applications p-type growth temperature is governed by annealing of active region, and optimization of $\mathrm{Mg}$-doped layer should be done for each wavelength.

\section{THE SAMPLE STRUCTURE}

The blue LED structures were produced together with Aston University, Ioffe Institute and CSTG partners. The magnesium is the only acceptor impurity practically used in MOCVD of GaN, and presence of hydrogen allows $\mathrm{Mg}$ to be incorporated on the c-plane at a reduced Mg flux [7]. As it was shown over five years ago, MOCVD carrier gas or $\mathrm{N}_{2} / \mathrm{H}_{2}$ gas mixture also can significantly affect $\mathrm{p}-\mathrm{GaN}$ surface morphology [6]. All of these observations led to the following investigations. The "sample A" (Z120227A) and "sample B" (Z120227B) were grown by MOVPE in AIX 2000 HT system on (0001) sapphire substrates. The Mg-doped region of these samples consists of AlGaN:Mg $(\sim 12-15 \mathrm{~nm})$ and GaN:Mg $(\sim 180-200 \mathrm{~nm})$. Growth temperature was $1010^{\circ} \mathrm{C}$ and reactor pressure $300 \mathrm{mBar}$. For both samples $\mathrm{AlGaN}$ growth was performed in $\mathrm{N}_{2}: \mathrm{H}_{2}=1: 1$ carrier gas mixture as it required by AlGaN growth conditions. GaN:Mg was grown in $\mathrm{N}_{2}: \mathrm{H}_{2}$ mixture or in $\mathrm{N}_{2}$ carrier gas. Due to slight changes in growth rate, growth time for $\mathrm{N}_{2}$ carrier gas was increased from 30 to 35 minutes to keep the same thickness, monitored by in-situ optical reflection. The $\mathrm{Mg}$ precursor flow was constant during growth $(280 \mathrm{sccm})$ resulting in $\mathrm{Mg}$ concentration by SIMS $\sim[\mathrm{Mg}] \sim 1 * 10^{20} \mathrm{~cm}^{-3}$ in $\mathrm{GaN}$ and $[\mathrm{Mg}] \sim 2 * 10^{20} \mathrm{~cm}^{-3}$ in AlGaN due to lower growth rate. After growth structures were annealed in-situ (without taking out of the reactor) at $865^{\circ} \mathrm{C}$ for 4 min in pure $\mathrm{N}_{2}$ ambient for $\mathrm{Mg}$ activation.

The structures (fig. 1) consists of an undoped $2 \mu \mathrm{m}$ GaN buffer layers, $2 \mu \mathrm{m}$ n-type GaN doped with silicon layers, following with the 12 period $1 \mathrm{~nm} \mathrm{InGaN/1nm} \mathrm{GaN} \mathrm{short} \mathrm{period} \mathrm{super} \mathrm{lattice} \mathrm{(SPSL),} \mathrm{20nm} \mathrm{GaN} \mathrm{layer,} \mathrm{3nm} \mathrm{InGaN}$ quantum well (QW), $8 \mathrm{~nm}$ GaN layer, 3nm InGaN QW, $5 \mathrm{~nm}$ GaN layer, $15 \mathrm{~nm}$ p-type AlGaN doped with magnesium electron blocking (EBL) layer and the top 200nm p-GaN layer doped with magnesium.

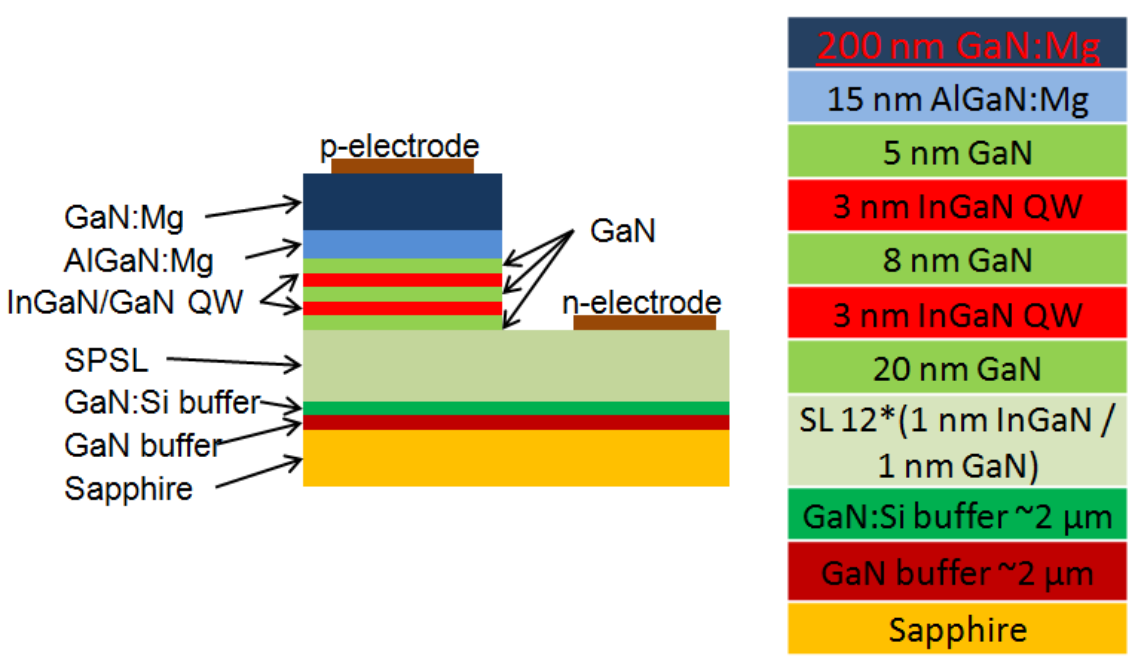

Figure 1. The blue LED device structure and epi-layer structure. 
The electrical contacts, p-electrode is made of $20 \mathrm{~nm} \mathrm{Ni} / 50 \mathrm{~nm} \mathrm{Au}$ annealed metals and n-electrode is $50 \mathrm{~nm} \mathrm{Ti} / 125 \mathrm{~nm}$ Pt/250nm Au based (fig. 2).

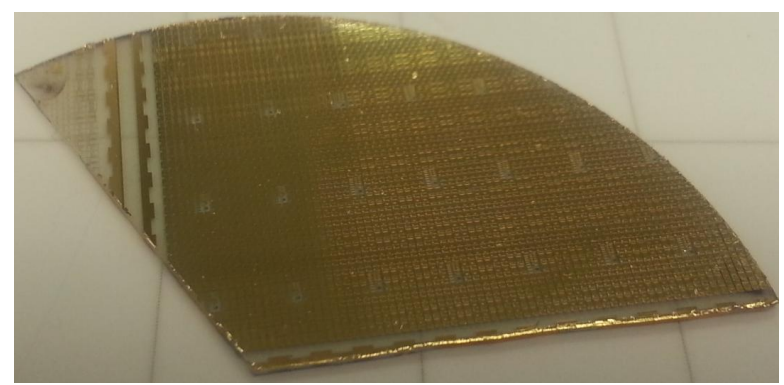

Figure 2. The wafer with the blue LED structures.

The produced blue LED devices has four different mesa sizes: 30x40

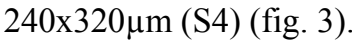

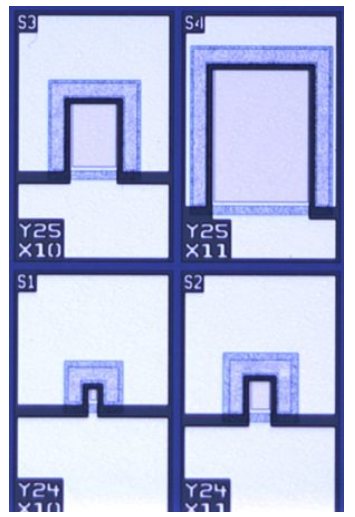

Figure 3. The different mesa sizes of blue LED devices.

\section{EXPERIMENT}

The blue LED devices were electrically and optically measured using Labsphere "CDS 600" spectrometer with LightMtrx software, Keithley 2400 power source, Keithely 4200 semiconductor characterization system, Signatone hybrid probe station H150W and Janis "CCS 450" cryostat system. The current-voltage (I-V), capacitance-voltage (CV), transfer length method (TLM), electro- and photo-luminescence techniques were applied (fig. 5).

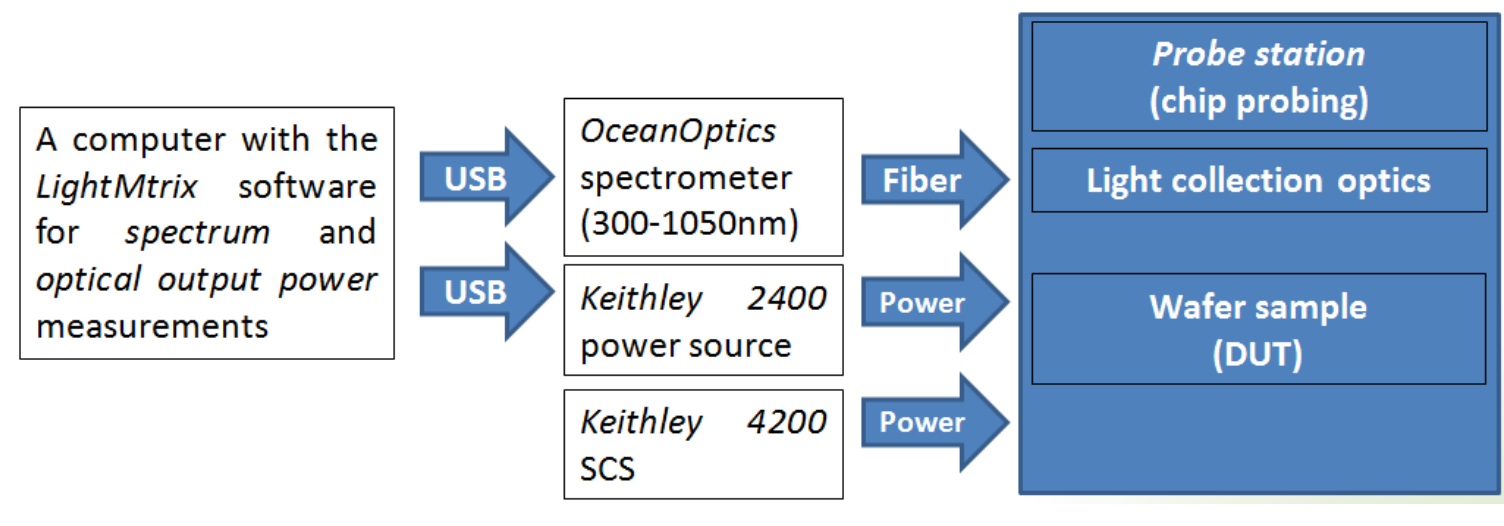

Figure 4. The blue LED measurement setup. 


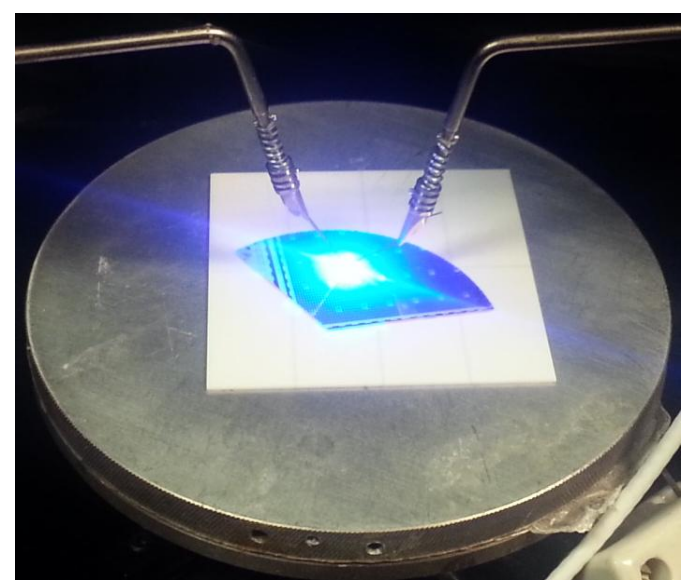

Figure 5. The blue LED device test in the probe station.

\section{RESULTS AND DISCUSSION}

\subsection{Current-voltage (I-V) characteristics}

The blue LEDs mesa size 240x320 $\mu \mathrm{m}$ (S4) samples "Z120227A" and "Z120227B" current-voltage characteristics were measured at room temperature (fig. 6). The Current-voltage characteristic shows that the sample "Z120227A" has lower voltage values than the sample "Z120227B". It is because of serial resistance and the influence of ideality factor. By applying the Shockley equation to the samples "Z120227A" and "Z120227B" measured I-V characteristic data, the ideality factor and series resistance were extracted (fig. 7) [8].

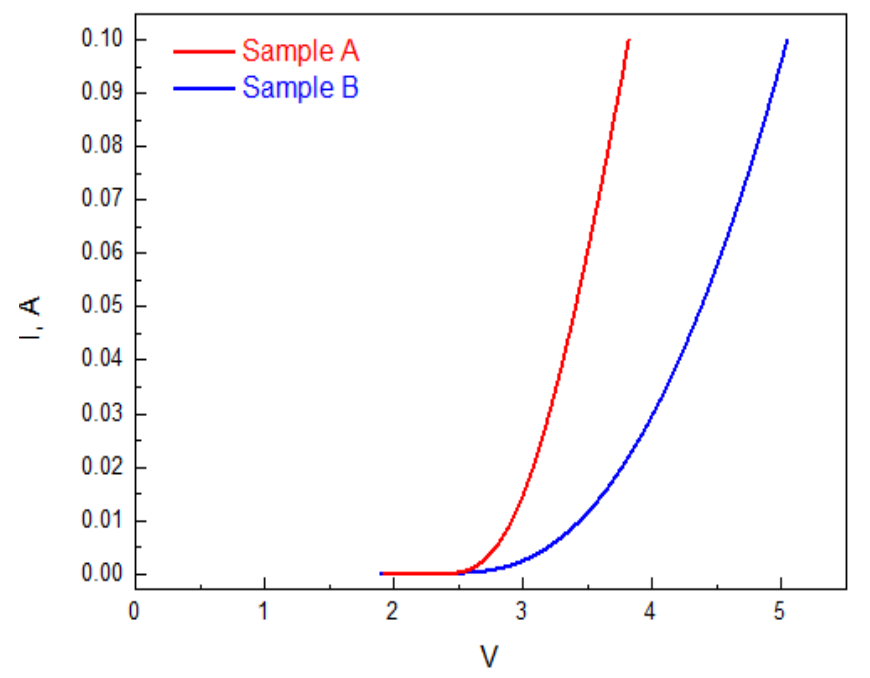

Figure 6. The blue LED samples, mesa size S4 I-V comparison. 


\begin{tabular}{|c|c|c|c|c|} 
Structure & \multicolumn{2}{|c|}{ A } & \multicolumn{2}{c|}{ B } \\
\hline Mesa size & $\beta$ & $\mathrm{R}_{\mathrm{s}}$ & $\beta$ & $\mathrm{R}_{\mathrm{S}}$ \\
\hline S1 & 8.2 & 17 & 10 & 26 \\
\hline S2 & 6.18 & 16 & 9 & 21 \\
\hline S3 & 5.48 & 8.5 & 6.46 & 17.5 \\
\hline S4 & 5 & 5 & 5.75 & 13.5 \\
\hline
\end{tabular}

Figure 7. The blue LED samples "Z120227A" and "Z120227B" extracted ideality factor and series resistances.

\subsection{Electro luminance characteristics}

The blue LED samples "Z120227A" and "Z120227B" electro-optical output intensities were measured at room temperature (fig. 8). The EL comparative measurement was performed for 4 different size contact pads and shows that the Z120227A - sample was more efficient. It was observed that the blue LED sample "Z120227A" mesa size S4 has twice higher intensity than sample "Z120227B" mesa size S4.

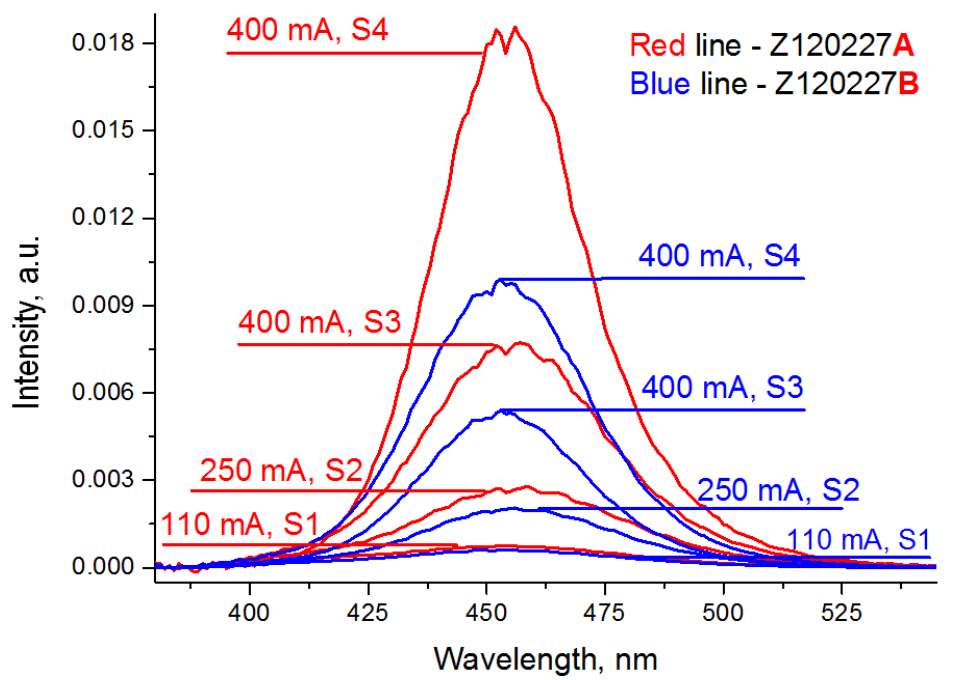

Figure 8. The blue LED samples "Z120227A" and "Z120227B" electro-optical output intensities compared.

\subsection{Photo luminance characteristics}

The blue LED samples "Z120227A" and "Z120227B" low temperature photoluminance measurement was performed (fig. 9). The blue LED samples were pumped using $405 \mathrm{~nm}$ wavelength laser diode producing $400 \mathrm{~mW}$ optical output power. It was noted that sample "Z120227A" mesa size S4 shows higher photo luminance intensity output than sample "B" mesa size S4. It suggest the less radiative centers in the sample "Z120227B" optical active regions. 


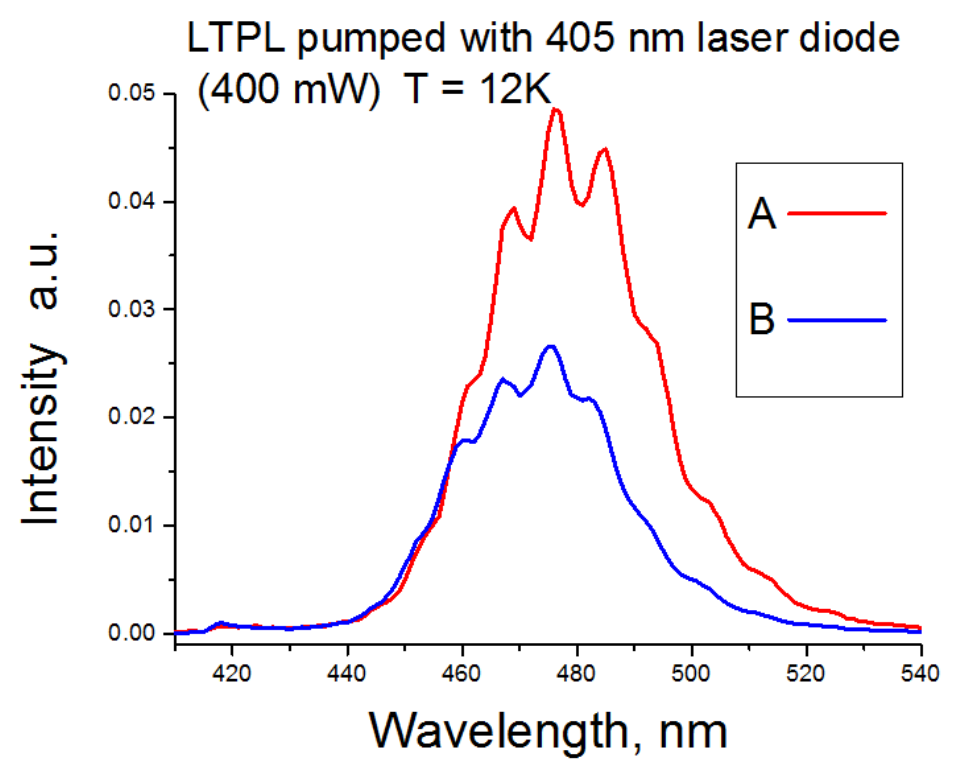

Figure 9. The blue LED samples "Z120227A" and "Z120227B" photo luminance intensities compared.

\subsection{Capacitance-voltage (C-V) characteristics}

Capacitance-voltage measurements were performed on the blue LED samples "Z120227A" and "Z120227B" mesa sizes S4 at room temperature (fig. 10). The "step" like behavior shows inhomogeneous distribution of charges in the depletion region. It could be associated with the defects in the epi-layers due to impure materials used.

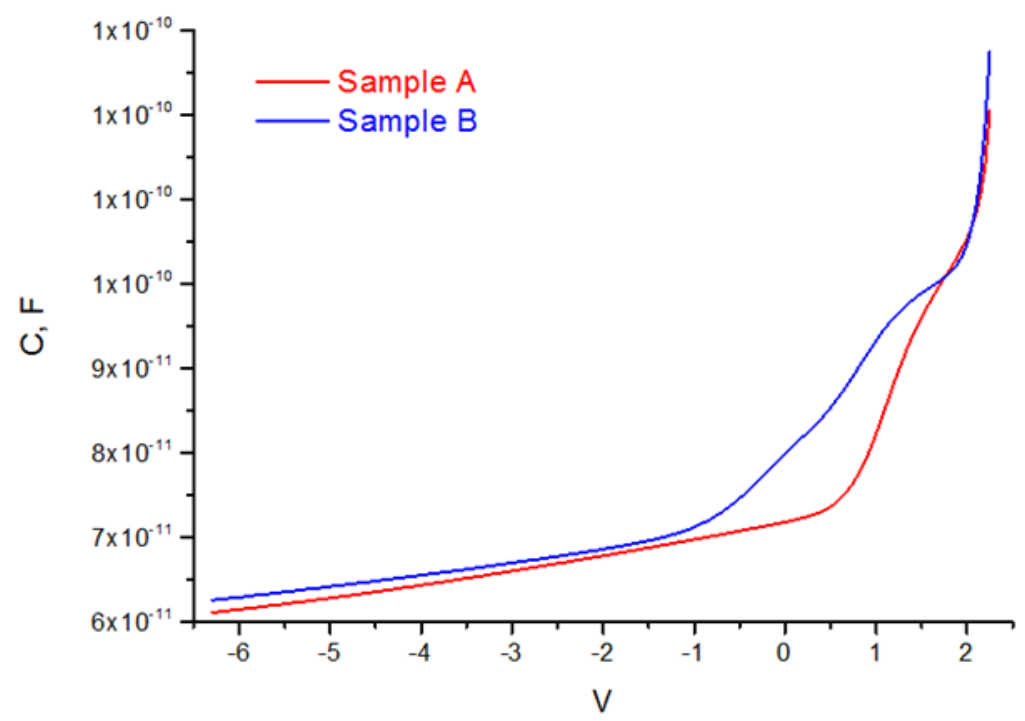

Figure 10. The blue LED samples "Z120227A" and "Z120227B" mesa size S4 capacitance measurement.

The apparent charge profile was obtained from the C-V measurement data (fig. 11) [9]. This charge can be attributed to electrically active $\mathrm{Mg}$ ions diffused to the active region or induced defects of another nature. 


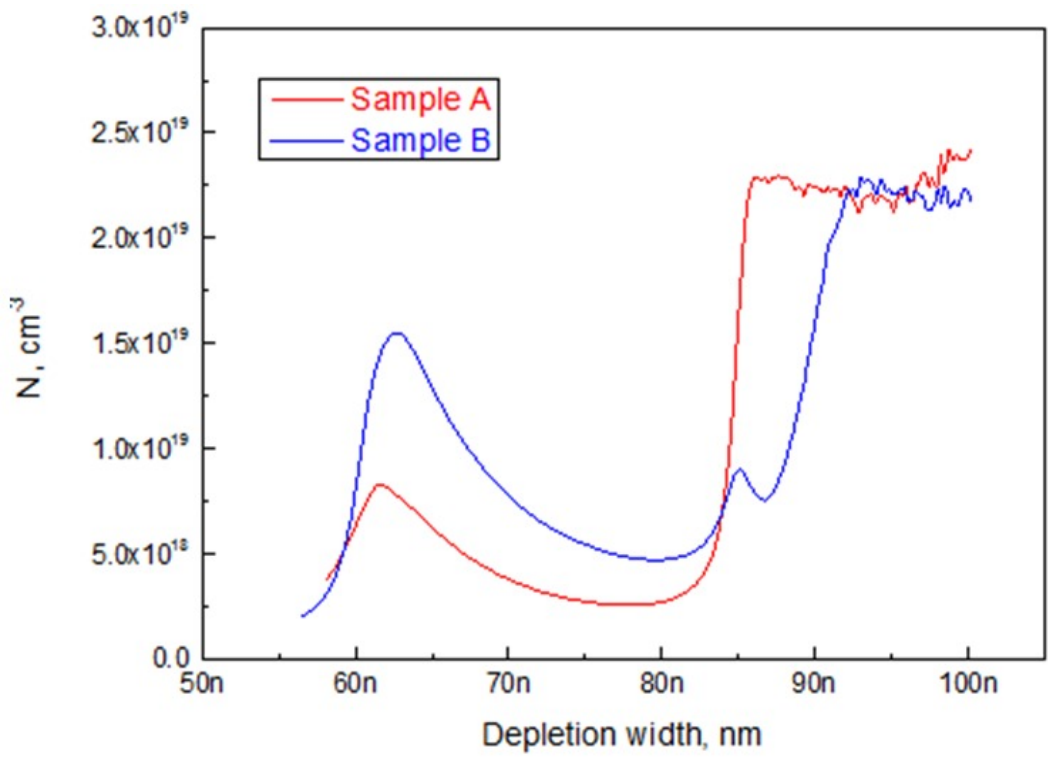

Figure 11. The blue LED samples "Z120227A" and "Z120227B" apparent charge profiles extracted.

\subsection{Transfer length method (TLM) characteristics}

Transfer length measurement were performed the blue LED samples "Z120227A" and "Z120227B" in four different places on the sample wafer (fig. 12). The dimensions of pads are 200 by $100 \mu \mathrm{m}$. The spacing are 10, 20, 30, 40, 50,60, $70 \mu \mathrm{m}$. It was measured and compared the top p-GaN layer sheet resistance (fig. 13). The blue LED sample "Z120227A" has much lower sheet resistance than sample "Z120227B".

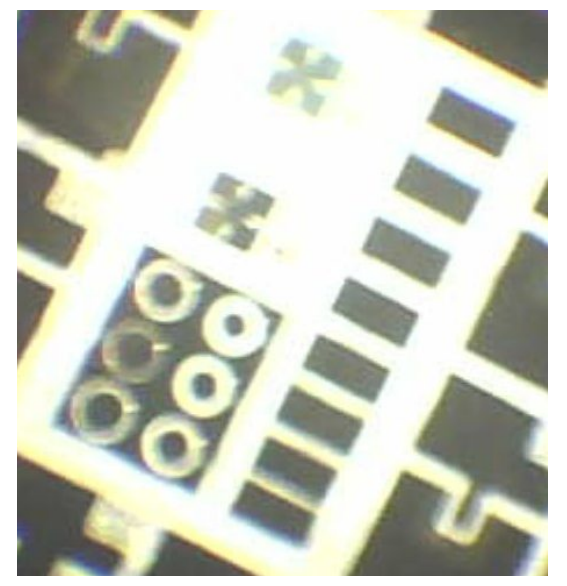

Figure 12. The blue LED samples "Z120227A" and "Z120227B" TLM measurements. 


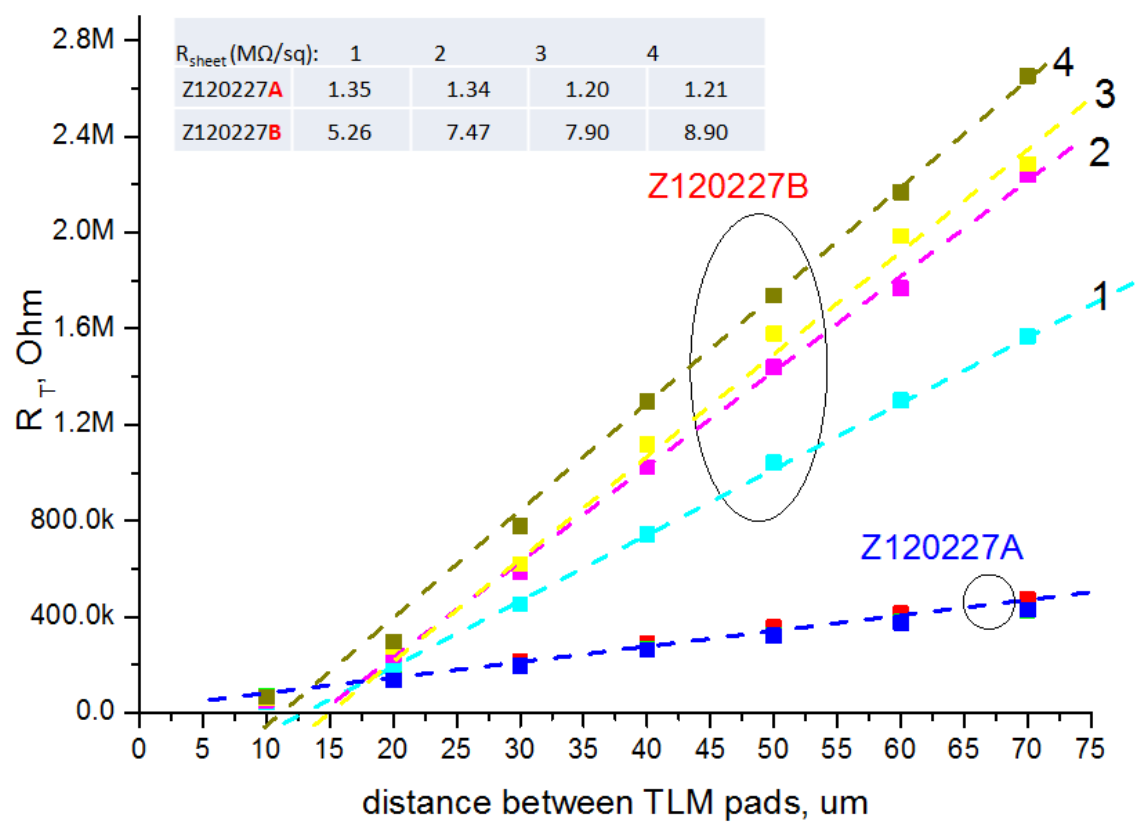

Figure 13. The blue LED samples "Z120227A" and "Z120227B" TLM measurements compared.

\section{CONCLUSION}

We investigated two blue LED structures frown in different growth environment with the main difference between the structures in the growth conditions of the top p-GAN epi-layers. The growing environments were varied due to the incorporation of Magnesium. In theory, $\mathrm{Mg}$ incorporates in GaN layer as shallow and as deep level (in $\mathrm{Mg}+\mathrm{H}$ complex) acceptor, resulting in $\mathrm{p}-\mathrm{GaN}$ conductivity compensation. The hydrogen environment improves the $\mathrm{Mg}$ incorporation rate, whlist $\mathrm{Mg}$ activation in $\mathrm{H}_{2}$ (annealing) strongly affects free hole concentration. So, the growth of $\mathrm{p}-\mathrm{GaN}$ in $\mathrm{N}_{2} / \mathrm{H}_{2}$ atmosphere increases deep level concentrations acting as hall traps (increasing resistance) and non-radiative centres (decreasing EL and PL). From comparative analysis of two blue LED structures, the advantage of the pure $\mathrm{N}_{2}$ growing environment was observed. The use of the pure $\mathrm{N}_{2}$ atmosphere during growth of p-type GaN improves serial resistance and EL intensity of LED and yields to higher efficiencies. The mixed growth atmosphere leds to increased series and sheet resistances of p-GaN layer. EL and PL measurements confirmed the advantage of the pure $\mathrm{N}_{2}$ utilization and $C\left(V_{R}\right)$ measurement pointed the increase of static charge concentration near the $\mathrm{p}-\mathrm{GaN}$ interface in the $\mathrm{B}$ structure.

\section{ACKNOWLEDGEMENTS}

This work was supported by the EU FP7 programme through the NEWLED project (contract no. 318388). 


\section{REFERENCES}

[1] Department of Energy, US, "SSL, R\&D Plan", May 2015: <energy.gov/sites/prod/files/2015/06/f22/ssl_rdplan_may2015_0.pdf>

[2] EC FP7 project NEWLED, "Nanostructured Efficient White LEDs", 2013: < http://www.newled-fp7.eu/>

[3] E. Fred Schubert, "Light emitting diodes", 2nd Edition, Cambridge University Press, p.353, 2006.

[4] Herbert Paul Maruska, Walden Clark Rhines, "A modern perspective on the history of semiconductor nitride blue light sources", Solid-State Electronics, Volume 111, September 2015, Pages 32-41.

[5] O. Svenska, S. Suihkonena, T. Langa, H. Lipsanena, M. Sopanena, M.A. Odnoblyudovb, V.E. Bougrovb, "Effect of growth conditions on electrical properties of Mg-doped p-GaN", Journal of Crystal Growth, Volume 298, January 2007, Pages 811-814.

[6] W. V. Lundin et.al., "Effect of carrier gas and doping profile on the surface morphology of MOVPE grown heavily doped GaN:Mg layers", Semiconductors, Vol. 43, No. 7, pp. 963-967, 2009.

[7] A. J. Ptak, et.al., "Magnesium incorporation in GaN grown by molecular-beam epitaxy", Appl. Phys. Lett. 78, $285,2001$.

[8] Jay M. Shah, Y.-L. Li, Th. Gessmann, and E. F. Schubert, "Experimental analysis and theoretical model for anomalously high ideality factors $(\mathrm{n}>2)$ in $\mathrm{AlGaN} / \mathrm{GaN}$ p-n junction diodes", JOURNAL OF APPLIED PHYSICS, VOLUME 94, NUMBER 415 AUGUST 2003.

[9] Y. Xia, E. Williams, Y. Park, I. Yilmaz, J.M. Shah, E.F. Schubert and C. Wetzel, "Discrete Steps in the Capacitance-Voltage Characteristics of GaInN/GaN Light Emitting Diode Structures", MRS Proceedings, 831, E3.38, PROC-831-E3.38., 2004. 